\title{
RJKBand Observations of the Optical Afterglow of GRB 991216
}

\section{Citation}

Garnavich, Peter M., Saurabh Jha, Michael A. Pahre, Krzysztof Z. Stanek, Robert P. Kirshner, Michael R. Garcia, Andrew H. Szentgyorgyi, and John L. Tonry. 2000. "RJKBand Observations of the Optical Afterglow of GRB 991216." The Astrophysical Journal 543 (1): 61-65. https:// doi.org/10.1086/317102.

\section{Permanent link}

http://nrs.harvard.edu/urn-3:HUL.InstRepos:41399854

\section{Terms of Use}

This article was downloaded from Harvard University's DASH repository, and is made available under the terms and conditions applicable to Other Posted Material, as set forth at http:// nrs.harvard.edu/urn-3:HUL.InstRepos:dash.current.terms-of-use\#LAA

\section{Share Your Story}

The Harvard community has made this article openly available. Please share how this access benefits you. Submit a story.

Accessibility 
The Astrophysical Journal, 543:61-65, 2000 November 1

(c) 2000. The American Astronomical Society. All rights reserved. Printed in U.S.A.

\title{
RJK BAND OBSERVATIONS OF THE OPTICAL AFTERGLOW OF GRB $991216^{1}$
}

\author{
Peter M. Garnavich \\ University of Notre Dame, Department of Physics, 225 Nieuwland Science Hall, Notre Dame, IN 46556; pgarnavi@nd.edu \\ Saurabh Jha, Michael A. Pahre, ${ }^{2}$ Krzysztof Z. Stanek, ${ }^{2}$ Robert P. Kirshner, \\ Michael R. GARCIA, AND ANDREW H. SZENTGYORGYI \\ Harvard-Smithsonian Center for Astrophysics, 60 Garden Street, Cambridge, MA 02138; sjha@cfa.harvard.edu, kstanek@cfa.harvard.edu, \\ mpahre@cfa.harvard.edu, mgarcia@cfa.harvard.edu, aszentgyorgyi@cfa.harvard.edu
}

\section{AND}

JOHN L. TONRY

University of Hawaii, Institute for Astronomy, 2680 Woodlawn Drive, Honolulu, HI 96822;jt@avidya.ifa.hawaii.edu Received 2000 March 29; accepted 2000 May 22

\begin{abstract}
We present near-infrared and optical observations of the afterglow to the gamma-ray burst 991216 obtained with the Fred L. Whipple Observatory $1.2 \mathrm{~m}$ telescope and the University of Hawaii $2.2 \mathrm{~m}$ telescope. The observations range from 15 hours to 3.8 days after the burst. The temporal behavior of the data is well described by a single power-law decay $t^{-1.36 \pm 0.04}$, independent of wavelength. The optical spectral energy distribution, which is corrected for significant Galactic reddening of $E(B-V)=0.626$, is well fitted by a single power law with $v^{-0.58 \pm 0.08}$. Combining the IR/optical observations with a Chandra $X$-Ray Observatory measurement gives a spectral index of $-0.8 \pm 0.1$ in the synchrotron cooling regime. A comparison between the spectral and temporal power-law indices suggests that a jet is a better match to the observations than a simple spherical shock.
\end{abstract}

Subject headings: gamma rays: bursts - shock waves

\section{INTRODUCTION}

The BeppoSAX (Boella et al. 1997) and Rossi X-Ray Timing Explorer (RXTE; Levine et al. 1996) satellites have brought a new dimension to gamma-ray burst (GRB) research by providing rapid localizations of several bursts per year. This has allowed many GRBs to be followed up at other wavelengths, ranging from the X-ray (Costa et al. 1997) and optical (van Paradijs et al. 1997) to the radio (Frail et al. 1997). Precise positions have also allowed redshifts to be measured for a number of GRBs (e.g., GRB 970508; Metzger et al. 1997), providing definitive proof of their cosmological origin.

The extremely bright GRB 991216 was detected by BATSE (Kippen, Preece, \& Giblin 1999) on December 16.671544 UT, with its peak flux (fluence) ranking it as the second (13th) of all BATSE bursts detected so far. The RXTE Proportional Counter Array search for the X-ray afterglow of GRB 991216 started about 4 hours after the burst (Takeshima et al. 1999) and detected a strong, decaying X-ray afterglow, providing a much improved burst position. It should be noted that the X-ray afterglow of GRB 991216 was also detected by the much less sensitive $R X T E$ all-sky monitor instrument as early as 1 hour after the burst (Corbet \& Smith 1999), providing a measurement of the X-ray afterglow at times that have previously not been studied. In addition, observations of GRB 991216 by the Chandra $X$-Ray Observatory resulted in the first X-ray afterglow with a position determined to arcsecond accuracy (Piro et al. 1999).

The optical afterglow of GRB 991216 was identified by Uglesich et al. (1999) with data taken about 12 hours after

\footnotetext{
${ }^{1}$ Based on the observations collected at the F. L. Whipple Observatory $1.2 \mathrm{~m}$ telescope and the University of Hawaii $2.2 \mathrm{~m}$ telescope.

${ }^{2}$ Hubble Fellow.
}

the burst (December 17.142 and 17.372 UT) using the MDM $1.3 \mathrm{~m}$ telescope. It was recognized as a bright variable object $(R \approx 18.8$ on December 17.142$)$ that was not present in the digitized POSS II plate and was declining with a temporal decay index of $\approx-1.4$. Numerous independent observations of the fading optical transient (OT) followed discovery, starting with Henden et al. (1999) and Jha et al. (1999). Near-infrared observations were also reported by Vreeswijk et al. (1999a) and Garnavich et al. (1999a).

Absorption lines at $z=1.02$, which are seen in the optical spectrum of GRB 991216 that was taken with the VLT Antu $8 \mathrm{~m}$ telescope by Vreeswijk et al. (1999b), provide a lower limit to the redshift of the GRB source. Given the gamma-ray fluence (Kippen 1999), the isotropic energy from the burst was more than $8 \times 10^{53} \mathrm{ergs}\left(H_{0}=65 \mathrm{~km}\right.$ $\mathrm{s}^{-1} \mathrm{Mpc}^{-1}, \Omega_{m}=0.3, \Omega_{\Lambda}=0.7$ ), or nearly half a solar rest mass radiated away in under $10 \mathrm{~s}$. This exceedingly large energy requirement can be reduced if the burst emission is beamed. To date evidence for jets has been found in only a handful of GRB afterglows (Sari, Piran, \& Halpern 1999; Kulkarni et al. 1999; Stanek et al. 1999), and it remains to be shown whether anisotropy is ubiquitous.

We present optical and near-IR photometry of GRB 991216 from observations obtained at the Hawaii $2.2 \mathrm{~m}$ and the Fred L. Whipple Observatory (FLWO) $1.2 \mathrm{~m}$ telescopes. We describe the data and the reduction procedure in $\S 2$. In $\S 3$ we discuss the multiband temporal behavior of the GRB OT. In $\S 4$ we describe the broadband spectral properties of the afterglow deduced from our IR/optical data.

\section{OBSERVATIONS}

The near-infrared data were obtained with the FLWO 1.2 meter telescope on four consecutive nights beginning 1999 December 17.22 (UT). Images were taken with the STELIRCam two-channel IR camera that utilizes two $256^{2}$ pixel $\mathrm{HgCdTe}$ arrays. A dichroic mirror splits the beam at 


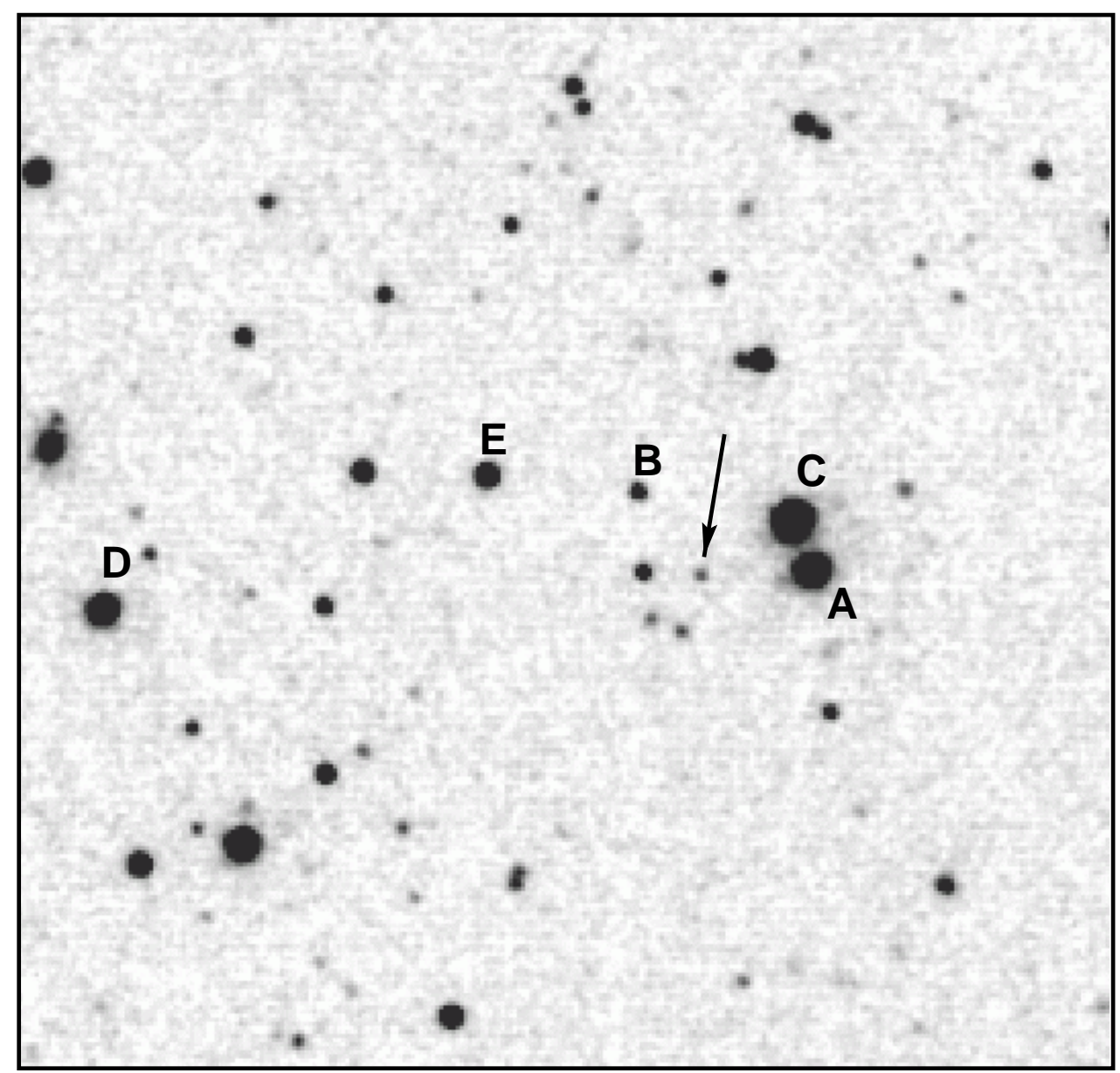

FIG. 1.-Finding chart for the field of GRB 991216 taken in the $J$ band. The optical transient is indicated with an arrow, and stars calibrated as secondary standards are shown using the convention of Jha et al. (1999). North is up, east to the left, and the field is approximately $3^{\prime}$ on a side.

$\lambda \approx 1.8 \mu \mathrm{m}$ allowing simultaneous observations in two filters. The GRB afterglow was observed in $J$ and $K$ filters manufactured by Barr. The camera has three sets of reimaging optics, and we employed the $5^{\prime}$ field of view with a $1^{\prime \prime} .2$ per pixel scale.

We immediately began imaging the $R X T E$ localization error box after being notified through the GCN Circulars of a bright GRB detected by BATSE. A $3 \times 3$ mapping $\left(15^{\prime}\right.$ field) around the initial $R X T E$ position was performed with two $60 \mathrm{~s}$ exposures taken at each pointing. A $J=17 \mathrm{mag}$ object that did not appear on the digitized sky survey was tentatively identified as the afterglow candidate (Garnavich et al. 1999b), however it was pointed out by Diercks et al. (1999b) that the star appeared on the POSS II N emulsion photographs and was likely to be a very red star. $R X T E$ revised its error box $8^{\prime}$ northward from the original position and a new mapping was begun. Uglesich et al. (1999) then identified the true afterglow near the revised position soon after observations at FLWO were terminated. Fortunately the original mapping and the mapping centered on the revised $R X T E$ error box included the object in several of the images. In subsequent nights GRB 991216 was observed in $J$ and $K$ with $9 \times 60 \mathrm{~s}$ exposure sets. An extensive number of Persson et al. (1998) standards were observed on December 18 (UT) and used to calibrate stars in the GRB field (Table 1; Fig. 1). Our $J$ - and $K$-filter calibrations are in good agreement with that of Henden, Guetter, \& Vrba (2000), but our GRB magnitudes (Table 2) are $20 \%-30 \%$ fainter in the $J$ filter and brighter in the $K$ filter than the Vreeswijk et al. (1999a) infrared photometry.

After the optical counterpart was identified a single exposure of the field was obtained with the University of Hawaii

TABLE 1

SECONDARY STANDARDS NEAR GRB 991216

\begin{tabular}{cccccc}
\hline \hline Star & R.A. (J2000) & Decl. (J2000) & $R$ & $J$ & $K$ \\
\hline A ..... & 050929.80 & 111708.4 & $15.38(02)$ & $13.34(03)$ & $12.52(03)$ \\
B ..... & 050932.14 & 111723.6 & $19.53(05)$ & $17.07(06)$ & $16.15(08)$ \\
C $\ldots . .$. & 050930.07 & 111718.3 & $\ldots$ & $12.60(03)$ & $11.80(03)$ \\
D ..... & 050939.29 & 111659.4 & $15.21(02)$ & $13.73(03)$ & $13.23(05)$ \\
E ..... & 050934.16 & 111726.5 & $18.43(04)$ & $15.19(05)$ & $14.14(06)$ \\
\hline
\end{tabular}


TABLE 2

GRB $991216 J$ - AND $K$-BAND Light CuRveS

\begin{tabular}{lcccc}
\hline \hline 1999 Dec UT & $\begin{array}{c}\text { Hours after } \\
\text { the Burst }\end{array}$ & $J$ & $K$ & $\begin{array}{c}\text { Exposure } \\
\text { (minutes) }\end{array}$ \\
\hline $17.22 \ldots \ldots \ldots$. & 13.2 & $16.75(06)$ & $15.01(08)$ & 2 \\
$17.40 \ldots \ldots \ldots$. & 17.5 & $17.07(06)$ & $15.29(05)$ & 8 \\
$18.25 \ldots \ldots \ldots$. & 37.9 & $18.11(07)$ & $16.54(07)$ & 9 \\
$18.37 \ldots \ldots \ldots$. & 40.8 & $18.45(08)$ & $16.71(08)$ & 9 \\
$19.30 \ldots \ldots \ldots$ & 63.1 & $18.90(10)$ & $17.17(25)$ & 18 \\
$20.28 \ldots \ldots \ldots$. & 86.6 & $19.46(13)$ & $>17.5$ & 25 \\
\hline
\end{tabular}

88 inch telescope. By then, the target was well past the meridian and the data suffered from a high air mass. On December 18 (UT) the field was observed at two epochs, and Landolt standards (Landolt 1992) were imaged to calibrate the data. Since GRB 991216 was only observed in the $R$ filter, no color correction was possible, and we estimate the uncertainty from the unknown color term as $5 \%$. Our $R$-band calibration of stars A and B are in good agreement with that found by Dolan et al. (1999). Our final $R$-band magnitudes (Table 3 ) are on average 0.08 mag fainter than the preliminary magnitudes given in Jha et al. (1999) and Garnavich et al. (1999a).

From our optical imaging, we find a position for the transient of $\alpha=05^{\mathrm{h}} 09^{\mathrm{m}} 31^{\mathrm{s}} \mathrm{s} 29, \delta=+11^{\circ} 17^{\prime} 07^{\prime \prime} .3(\mathrm{~J} 2000)$ with an accuracy of \pm 0 ".2 based on positions from the United States Naval Observatory A2.0 catalog (Monet et al. 1996).

\section{THE TEMPORAL BEHAVIOR}

Figure 2 shows the $R$-, $J$-, and $K$-band light curves of GRB 991216. Additional $R$-band points obtained from GCN Circulars (Uglesich et al. 1999; Dolan et al. 1999; Vreeswijk et al. 1999a; Diercks et al. 1999a; Jensen et al. 1999; Leibowitz et al. 1999; Mattox 1999) are also plotted, but the comparison stars used in their calibration are sometimes not known, and these points are here used only to confirm the trends seen in our data. Late-time observations by Djorgovski et al. (1999) and Schaefer (2000) use the Dolan et al. (1999) or Jha et al. (1999) calibrations and are consistent with our points. The light curves appear to follow a single power law between 0.5 days and 4 days after the burst. So as not to confuse the temporal and spectral variations, we will use the convention that $F_{v} \propto t^{-\alpha} v^{-\beta}$.

A single power law was fitted to our data points by allowing the magnitude shift between the $J$ and $K$ bands and the shift between the $J$ and $R$ bands to be free parameters. The result is shown as the solid lines in Figure 2 and provides an index of $\alpha=1.36 \pm 0.04(1 \sigma)$. Fitting the individual bands gives indices of $\alpha=1.44 \pm 0.06$ for the $K$ band, $1.31 \pm 0.06$ for the $J$ band, and $1.42 \pm 0.16$ for the $R$ band. Combining our three $R$-band observations with six observations from the GCN Circulars that were obtained within 4 days of the burst gives a power-law index of $\alpha=1.30 \pm 0.05$, which is somewhat steeper than the decay rate found by Sagar et al.

TABLE 3

GRB 991216 R-BAND Light CURVE

\begin{tabular}{lcc}
\hline \hline 1999 Dec UT & Hours after the Burst & $R$ \\
\hline $17.61 \ldots \ldots \ldots$. & 22.5 & $19.56(10)$ \\
$18.32 \ldots \ldots \ldots$. & 39.6 & $20.41(05)$ \\
$18.56 \ldots \ldots \ldots$ & 45.3 & $20.64(05)$ \\
\hline
\end{tabular}

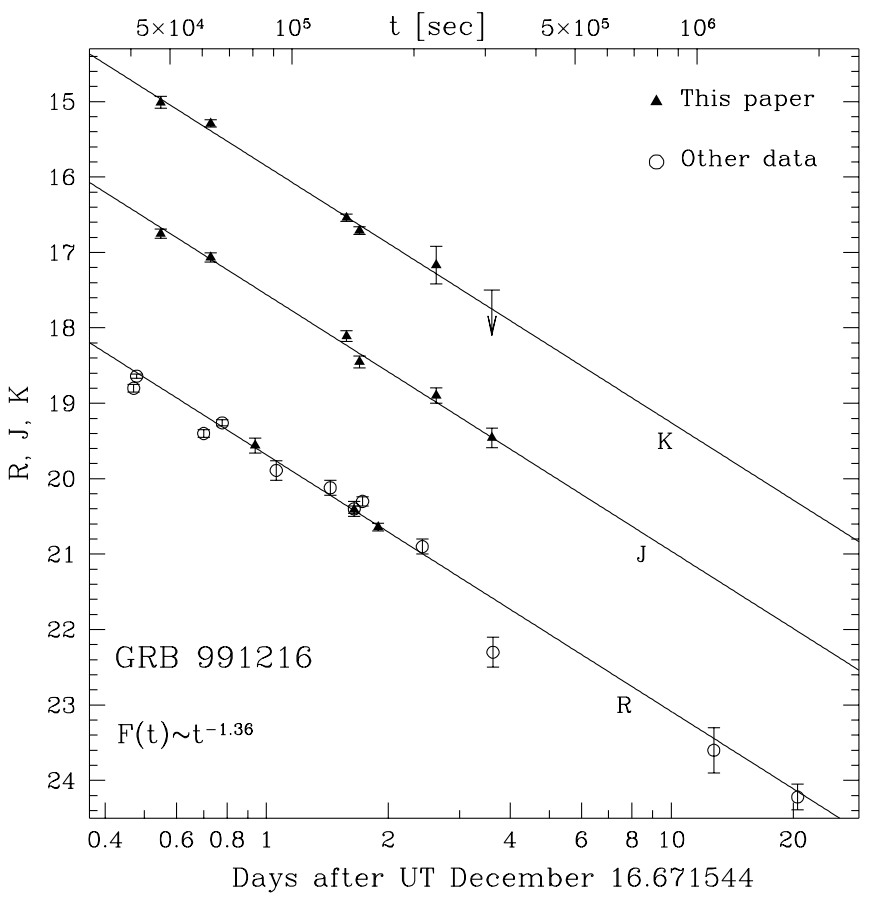

FIG. 2.-RJK light curves of GRB 991216. Our data is shown with solid points and other observations published in the GCN Circulars are shown as open points. Also shown is the single power-law fit obtained by combining all of our data.

(2000) from the raw GCN $R$-band magnitudes. Clearly a power-law index of $\alpha=1.36$ is a good fit to all three bands given the estimated errors. Extrapolating the $R$-band fit to the late-time observations by Schaefer (2000) and Djorgovski et al. (1999) shows that the single power law is consistent with the data out to 20 days after the burst. The $R$-band point by Mattox (1999) appears significantly below the trend. Our $J$-band photometry, obtained near that time, shows no deviation from the fit, however, we cannot rule out a change in slope beginning 4 days after the burst and then a recovery at late times because of a possible underlying supernova or host galaxy.

\section{REDDENING AND BROADBAND SPECTRAL ENERGY DISTRIBUTION}

GRB 991216 is located at Galactic coordinates of $l=$ $190.44, b=-16.63$. To remove the effects of the Galactic interstellar extinction we used the reddening map of Schlegel, Finkbeiner, \& Davis (1998, hereafter SFD). The expected Galactic reddening toward the burst is substantial, $E(B-V)=0.626 \mathrm{mag}$. We use $R_{V}=3.1$ and the standard reddening curve of Cardelli, Clayton, \& Mathis (1989), as tabulated by SFD (their Table 6), to correct our optical and IR data. As discussed by Stanek et al. (1999), there is some indication that the SFD map overestimates the $E(B-V)$ values by a factor of $1.3-1.5$ close to the Galactic plane $\left(|b|<5^{\circ}\right)$ and in high-extinction $\left(A_{V}>0.5 \mathrm{mag}\right)$ regions (Arce \& Goodman 1999). It is not clear at all that such a correction should be applied to the SFD $E(B-V)$ value for GRB 991216, but it would reduce this value to about $E(B-V)=0.46$.

We synthesize the optical/IR spectrum from our data by interpolating the magnitudes to a common time. As discussed in the previous section, the colors of GRB 991216 
counterpart do not show significant variation. We therefore select an epoch of December 18.32 UT (40 hours after the burst) for the color analysis, which is near the time when simultaneous $R J K$ data were taken.

We convert the $R J K$ magnitudes to fluxes using the effective wavelengths and normalizations of Fukugita, Shimasaku, \& Ichikawa (1995) for the optical observations and Mégessier (1995) for the IR observations. These conversions are accurate to about $5 \%$, which increases the error bars correspondingly. Note that while the error in the $E(B-V)$ reddening value has not been applied to the error bars of individual points, we include it in the error budget of the fitted slope. The results are plotted in Figure 3 for both the observed and the dereddened magnitudes. The corrected spectrum is well fitted by a single power law with $\beta=0.58 \pm 0.08$. If we use the lower value of $E(B-V)=0.46$, as discussed above, the corresponding number is $\beta=0.87 \pm 0.08$. We have assumed that there is no extinction within the host galaxy, but any reddening from the host will make the intrinsic spectrum more flat and reduce the derived value of $\beta$.

A radio observation (Taylor \& Berger 1999; Frail et al. 2000) found the afterglow at $8.5 \mathrm{GHz}$ to be $960 \pm 67 \mu \mathrm{Jy}$ on 1999 December 18.16. Adjusting our $K$-band flux to this date, we find a power-law index between the radio and near-IR observations to be -0.15 , much more shallow than the index between the near-IR and the optical observations. The Chandra X-Ray Observatory also observed the GRB on December 18.2 (UT) (Piro et al. 1999), and by converting to a flux density we find a power-law index between the IR and $\mathrm{X}$-ray points of $\beta=0.8 \pm 0.1$, which is slightly steeper than a simple extrapolation from the IR/optical data. We note that this slope is close to what is found for the IR/optical data if the extinction is set to the lower value of the range discussed above. Figure 4 shows the overall spectrum from the radio to the $\mathrm{X}$-rays and evidence for a spectra break at frequencies less than the $K$ band.

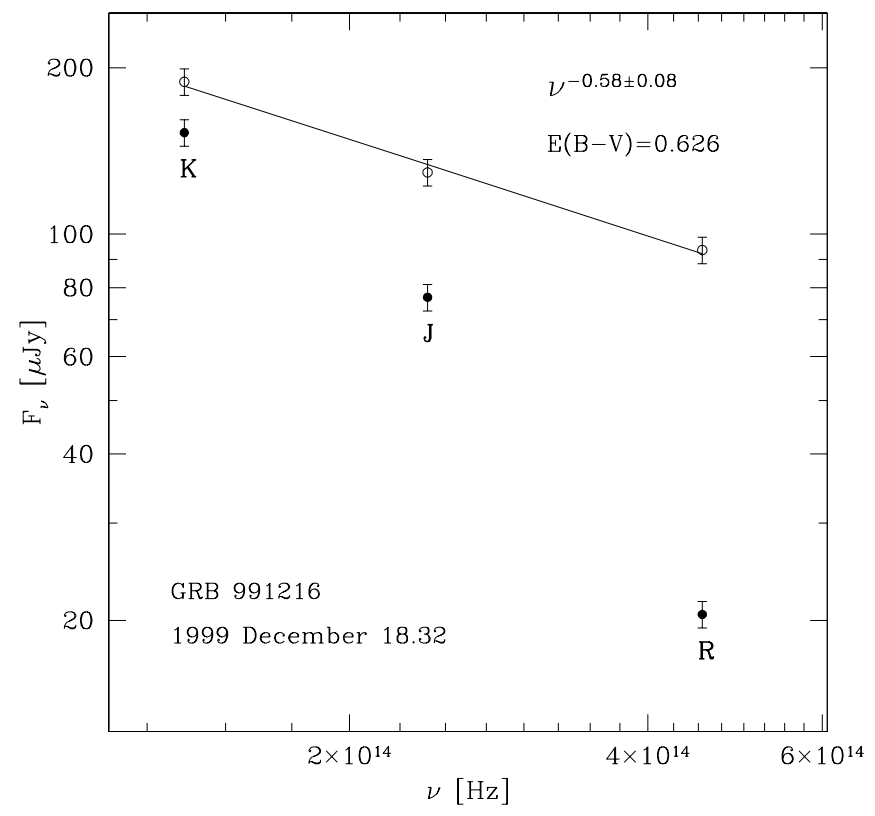

FIG. 3.-Synthetic spectrum of GRB 99121640 hours after the burst. The points at the top show the data corrected for a reddening of $E(B-V)=0.626 \mathrm{mag}$.

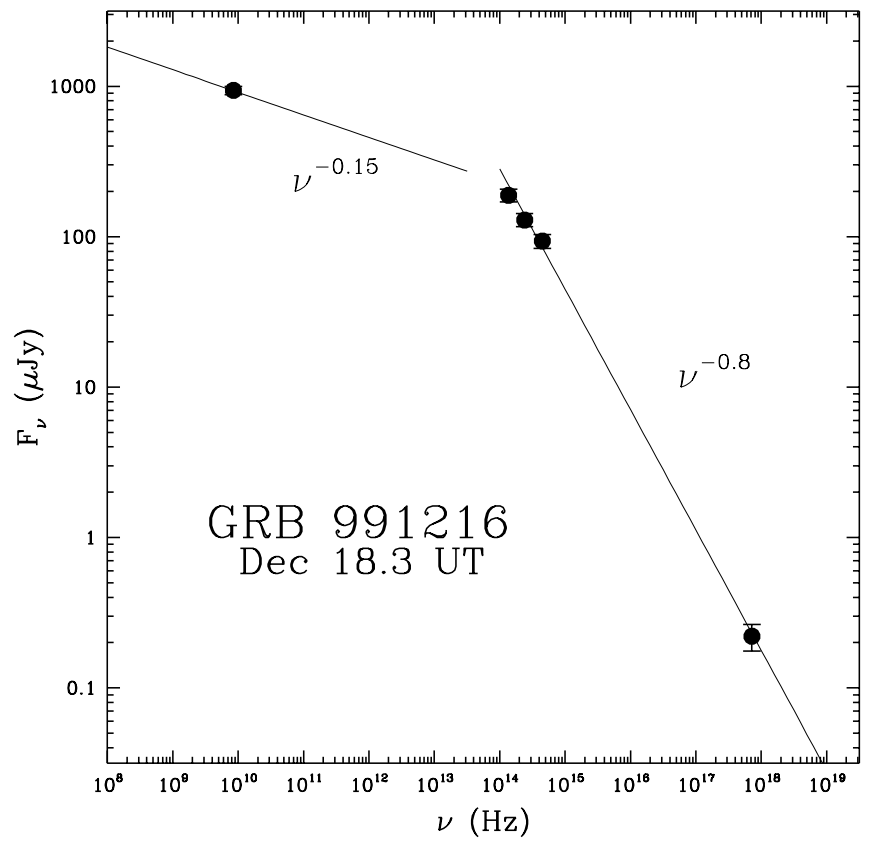

FIG. 4.- Spectrum of GRB 991216 over 10 orders of magnitude in frequency on December 18.3 (UT). The radio data are from Taylor \& Berger (1999) and Frail et al. (2000). The X-ray point is derived from Piro et al. (1999).

The GRB afterglow model described by Sari, Piran, \& Halpern (1999) can be used to compare the spectral and temporal power-law indices observed. The IR/optical region is within the cooling regime (Fig. 4), so that the observed spectral slope of $\beta=0.6$ ( 0.8 for IR to X-ray) gives an estimate of the electron distribution index of $p=1.2$ (1.6). For a spherical shock, we then expect a temporal index of $\alpha=(3 \beta-1) / 2=0.4(0.7)$, which is much more shallow than the observed index. For a jet, however, the expected light-curve index is $\alpha=2 \beta=1.2$ (1.6),which is close to the observed value of 1.4. At frequencies less than the cooling break, we expect a spectral index of 0.1 based on the IR/ optical slope. This is similar to the observed index of 0.15 , but it should be noted that other spectral breaks may be present between the radio and IR points.

\section{CONCLUSIONS}

We present well calibrated $R J K$ observations of GRB 991216. Our data indicates that the decay of the optical afterglow is well represented by a single power law with index $\alpha=1.36 \pm 0.04$ from 0.5 days to 4 days after the burst. Combining published late-time $R$-band observations with our data suggests a single power law is a good fit up to 20 days after the burst.

The optical spectral energy distribution corrected for significant Galactic reddening is well fitted by a single power law with an index of $\beta=0.58 \pm 0.08$. However, when the possible systematic error in the SFD extinction map is considered, the index may be somewhat steeper $(\beta=0.87 \pm 0.08)$. A Chandra X-ray observation obtained near the time of our photometry provides a spectral index between the near-IR and X-rays of $\beta=0.8 \pm 0.1$.

A comparison between the spectral and temporal powerlaw indices suggest that the GRB is not consistent with a 
simple spherical shock model. The IR/optical light curve and colors are better matched by a shock produced from a collimated jet.

S. Barthelmy, the organizer of the GRB Coordinates Network (GCN), is recognized for his extremely useful effort. Support for M. A. P. (HF-01099.01-97A) and K. Z. S.
(HF-01124.01-99A) was provided by NASA through Hubble Fellowship grants from the Space Telescope Science Institute, which is operated by the Association of Universities for Research in Astronomy, Inc., under NASA contract NAS 5-26555. R. P. K. and S. J. acknowledge NSF support through AST 98-19825 and a NSF Graduate Research Fellowship.
Arce, H. G., \& Goodman, A. A. 1999, ApJ, 512, L135

Boella, G., et al. 1997, A\&AS, 122, 299

Cardelli, J. A., Clayton, G. C., \& Mathis, J. S. 1989, ApJ, 345, 245

Corbet, R., \& Smith, D. 1999, GCN Circ. 506 (http://gcn.gsfc.nasa.gov/ $\mathrm{gcn} / \mathrm{gcn} 3 / 506 . \mathrm{gcn} 3)$

Costa, E., et al. 1997, Nature, 387, 783

Diercks, A., Bloom, J. S., Djorgovski, S. G., Kulkarni, S. R., Soifer, B. T Thompson, D. J., Gal, R. R., \& Mahabal, A. 1999a, GCN Circ. 497 (http://gcn.gsfc.nasa.gov/gcn/gcn3/497.gcn3)

Diercks, A., Djorgovski, S. G., Bloom, J. S., Mahabal, A., \& Gal, R. 1999b, GCN Circ. 470 (http://gcn.gsfc.nasa.gov/gcn/gcn3/470.gcn3)

Djorgovski, S. G., Goodrich, R., Kulkarni, S., Bloom, J., Diercks, A., Harrison, F., \& Frail, D. 1999, GCN Circ. 510 (http://gcn.gsfc.nasa.gov/gcn/ $\mathrm{gcn} 3 / \mathrm{gcn} 510 . \mathrm{gcn} 3)$

Dolan, C., Dell'Antonio, I., Jannuzi, B., \& Rhoads, J. 1999, GCN Circ. 486 (http://gcn.gsfc.nasa.gov/gcn/gcn3/486.gcn3)

Frail, D. A., et al. 2000, ApJ, sumbitted (astro-ph/0003138)

Frail, D. A., Kulkarni, S. R., Nicastro, L., Feroci, M., \& Taylor, G. B. 1997, Nature, 389, 261

Fukugita, M., Shimasaku, K., \& Ichikawa, T. 1995, PASP, 107, 945

Garnavich, P., Jha, S., Stanek, K. Z., Pahre, M., Garcia, M., Szentgyorgyi, A., \& Tonry, J. 1999a, GCN Circ. 495 (http://gcn.gsfc.nasa.gov/gcn/ gcn3/495.gcn3)

Garnavich, P., Stanek, K. Z., Garcia, M., Jha, S., \& Szentgyorgyi, A. 1999b, GCN Circ. 469 (http://gcn.gsfc.nasa.gov/gcn/gcn3/469.gcn3)

Henden, A., Guetter, H., \& Vrba, F. 2000, GCN Circ. 518 (http:// gcn.gsfc.nasa.gov/gcn/gcn3/518.gcn3)

Henden, A., et al. 1999, GCN Circ. 473 (http://gcn.gsfc.nasa.gov/gcn/gcn3/ 473.gcn 3 )

Jensen, B. L., et al. 1999, GCN Circ. 498 (http://gcn.gsfc.nasa.gov/gcn/ gen3/498.gcn3)

Jha, S., Kirshner, R., Stanek, K. Z., Garnavich, P., Garcia, M., Szentgyorgyi, A., \& Tonry, J. 1999, GCN Circ. 476 (http://gcn.gsfc.nasa.gov/gcn/ gcn3/476.gcn3)

Kippen, R. M. 1999, GCN Circ. 504 (http://gcn.gsfc.nasa.gov/gcn/gcn3/ 504.gcn3)

Kippen, R. M., Preece, R. D., \& Giblin, T. 1999, GCN Circ. 463 (http:// gcn.gsfc.nasa.gov/gcn/gcn3/463.gcn3)

\section{REFERENCES}

Kulkarni, S. R., et al. 1999, Nature, 398, 389

Landolt, A. U. 1992, AJ, 104, 340

Leibowitz, E. M., et al. 1999, GCN Circ. 499 (http://gcn.gsfc.nasa.gov/gcn/ gcn3/499.gcn3)

Levine, A. M., Bradt, H., Cui, W., Jernigan, J. G., Morgan, E. H., Remillard, R., Shirey, R. E., \& Smith, D. A. 1996, ApJ, 469, L33

Mattox, J. 1999, GCN Circ. 503 (http://gcn.gsfc.nasa.gov/gcn/gcn3/ 503.gcn 3 )

Mégessier, C. 1995, A\&A, 296, 771

Metzger, M. R., et al. 1997, Nature, 387, 879

Monet, D., et al. 1996, CD-ROM, USNO-SA2.0 Catalog (Washington, DC: USNO)

Persson, S. E., Murphy, D. C., Krzeminski, W., Roth, M., \& Rieke, M. J. 1998, AJ, 116, 2475

Piro, L., Garmire, G., Garcia, M., Marshall, F., \& Takeshima, T. 1999, GCN Circ. 500 (http://gcn.gsfc.nasa.gov/gcn/gcn3/500.gcn3)

Sagar, R., Mohan, V., Pandey, A. K., Pandey, S. B., \& Castro-Tirando, A. J. 2000, Bull. Astron. Soc. India, 28, 429

Sari, R., Piran, T., \& Halpern, J. 1999, ApJ, 519, L17

Schaefer, B. E. 2000, GCN Circ. 517 (http://gcn.gsfc.nasa.gov/gcn/gcn3/ 517.gcn 3)

Schlegel, D. J., Finkbeiner, D. P., \& Davis, M. 1998, ApJ, 500, 525

Stanek, K. Z., Garnavich, P. M., Kaluzny, J., Pych, W., \& Thompson, I. 1999, ApJ, 522, L39

Takeshima, T., Markwardt, C., Marshall, F., Giblin, T., \& Kippen, R. M. 1999, GCN Circ. 478 (http://gcn.gsfc.nasa.gov/gcn/gcn3/478.gcn3)

Taylor, G. B., \& Berger, E. 1999, GCN Circ. 483 (http://gcn.gsfc.nasa.gov/ $\mathrm{gcn} / \mathrm{gcn} 3 / 483 . \mathrm{gcn} 3$

Uglesich, R., Mirabal, N., Halpern, J., Kassin, S., \& Novati, S. 1999, GCN Circ. 472 (http://gcn.gsfc.nasa.gov/gcn/gcn3/472.gcn3)

van Paradijs, J., et al. 1997, Nature, 386, 686

Vreeswijk, P. M., Rol, E., Kouveliotou, C., Pian, E., Castro-Tirado, A., Pedersen, H., \& Greiner, J. 1999a, GCN Circ. 492 (http:// gcn.gsfc.nasa.gov/gcn/gcn3/492.gcn3)

Vreeswijk, P. M., et al. 1999b, GCN Circ. 496 (http://gcn.gsfc.nasa.gov/ $\mathrm{gcn} / \mathrm{gcn} 3 / 496 . \mathrm{gcn} 3$ ) 